\title{
A!
}

This is an electronic reprint of the original article.

This reprint may differ from the original in pagination and typographic detail.

Nguyen, H.Q.; Meschke, M.; Courtois, H; Pekola, J.P.

\section{Sub-50-mK Electronic Cooling with Large-Area Superconducting Tunnel Junctions}

Published in:

Physical Review Applied

DOI:

10.1103/PhysRevApplied.2.054001

Published: 04/11/2014

Document Version

Publisher's PDF, also known as Version of record

Please cite the original version:

Nguyen, H. Q., Meschke, M., Courtois, H., \& Pekola, J. P. (2014). Sub-50-mK Electronic Cooling with LargeArea Superconducting Tunnel Junctions. Physical Review Applied, 2(5), 1-6. [054001].

https://doi.org/10.1103/PhysRevApplied.2.054001

This material is protected by copyright and other intellectual property rights, and duplication or sale of all or part of any of the repository collections is not permitted, except that material may be duplicated by you for your research use or educational purposes in electronic or print form. You must obtain permission for any other use. Electronic or print copies may not be offered, whether for sale or otherwise to anyone who is not an authorised user. 


\title{
Sub-50-mK Electronic Cooling with Large-Area Superconducting Tunnel Junctions
}

\author{
H. Q. Nguyen, ${ }^{1,2, *}$ M. Meschke, ${ }^{1}$ H. Courtois, ${ }^{3,4}$ and J. P. Pekola ${ }^{1}$ \\ ${ }^{1}$ Low Temperature Laboratory (OVLL), Aalto University School of Science, \\ P.O. Box 13500, 00076 Aalto, Finland \\ ${ }^{2}$ Nano and Energy Center, Hanoi University of Science, VNU, Hanoi, Vietnam \\ ${ }^{3}$ Université Grenoble Alpes, Institut Néel, F-38042 Grenoble, France \\ ${ }^{4}$ CNRS, Institut Néel, F-38042 Grenoble, France
}

(Received 24 February 2014; revised manuscript received 19 April 2014; published 4 November 2014)

\begin{abstract}
In electronic cooling with superconducting tunnel junctions, the cooling power is counterbalanced by the interaction with phonons and by the heat flow from the overheated leads. We study aluminum-based coolers that are equipped with a suspended normal metal and an efficient quasiparticle drain. At intermediate temperatures, the phonon bath of the suspended normal metal is cooled. By adjusting the junction transparency, we control the injection current and, thus, the temperature of the superconducting lead at the optimum cooling point. The best device shows remarkable cooling from $150 \mathrm{mK}$ down to about $30 \mathrm{mK}$, a factor of 5 in temperature at a power of $40 \mathrm{pW}$. We discuss heat transport in our device and the reasons for cooling saturation at the low-temperature end.
\end{abstract}

DOI: 10.1103/PhysRevApplied.2.054001

\section{INTRODUCTION}

When a normal-metal-insulator-superconductor $(N-I-S)$ junction is biased near the superconducting gap, hot electrons tunnel into the superconductor and the normal metal is cooled down [1-6]. Based on this effect, micron-size electronic coolers attached to a dielectric platform [7] can cool an external object at temperatures well below $300 \mathrm{mK}$, a regime that traditionally belongs to macroscopic cryostats, such as adiabatic demagnetization or ${ }^{3} \mathrm{He}-{ }^{4} \mathrm{He}$ dilution refrigerators. In the best demonstration to date [8], a superconducting transition edge sensor has been cooled on a platform attached to $N-I-S$ junctions from 300 to $200 \mathrm{mK}$. Clearly, such a platform is of great interest as an alternative method to bring ultrasensitive low-temperature detectors [9], including those at the frontier of astronomical studies of dark matter [10], neutrinos [11], or the cosmic microwave background [12], into their proper working temperature. It allows those devices to work from a bath temperature higher than their own critical temperatures. Moreover, for space-borne applications [13], N-I-S coolers could reduce the payload noticeably. The ability to reach the sub-50-mK regime with $N-I-S$ coolers is crucial in order to open up new possibilities to cool qubits [14] and nanomechanical resonators [15] to their quantum ground states or to evacuate heat in electron-pumping devices [16]. This fact extends to the next generation of ultralow-temperature detectors, like superconducting quantum interference proximity transistor sensors [17] that are even more sensitive once operated in the sub-50-mK temperature regime.

To cool well, the normal metal needs to be isolated from the environment, and, at the same time, the hot

\footnotetext{
*hung@1tl.tkk.fi
}

superconducting leads need to be thermalized with the surrounding bath. In the normal metal, electrons interact with the lattice phonons, with a coupling strength decaying quickly towards low temperatures as $T^{5}$. Under some conditions, the lattice phonons can decouple from the thermal bath of the substrate phonons [18-20]. In the superconducting leads, hot quasiparticles at an energy just above the energy gap are generated $[3,4,21]$. It is a challenge to thermalize them, as quasiparticle relaxation rates decrease exponentially with lowering the temperature. Typically, these hot particles can be evacuated to a quasiparticle trap, which is a layer of normal metal in close contact with the superconductor [22-24].

When assuming that the electronic populations in both the normal metal and the superconductor can be described by Fermi distributions at respective temperatures $T_{N}$ and $T_{S}$, the cooling power of a $N-I-S$ junction at its optimum cooling bias $e V \simeq \Delta-0.66 k_{B} T_{N}$ is given by [3]

$\dot{Q}_{N I S} \simeq \frac{\Delta^{2}}{e^{2} R_{T}}\left[0.59\left(\frac{k_{B} T_{N}}{\Delta}\right)^{3 / 2}-\sqrt{\frac{2 \pi k_{B} T_{S}}{\Delta}} e^{-\Delta / k_{B} T_{S}}\right]$.

Here, $R_{T}$ is the tunnel resistance, $\Delta$ is the superconducting gap, $k_{B}$ is the Boltzmann constant, and $e$ is the electron charge. If the superconducting lead is not properly thermalized so that $T_{S}\left(>T_{\text {bath }}\right)$ approaches the superconducting transition temperature $T_{c}$, the term $\exp \left(-\Delta / k_{B} T_{S}\right)$ becomes significant and $\dot{Q}_{N I S}$ diminishes. In many cases, one assumes no overheating, and at low temperature one can neglect the second term; thus, $\dot{Q}_{N I S} \propto T_{N}^{3 / 2}$. In the lowtemperature regime $T_{N} \ll T_{c}$, the efficiency of the cooler is then given by [3] 


$$
\eta=\frac{\dot{Q}_{N I S}}{I V} \simeq 0.7 \frac{T_{N}}{T_{c}}
$$

It amounts to about $20 \%$ near $T_{N}=350 \mathrm{mK}$ for aluminum, which is the standard choice of a superconductor. In general, the most significant opposing heat current to $\dot{Q}_{N I S}$ comes from the electron-phonon interaction in the normal metal. The most accepted form for a metal is written

$$
\dot{Q}_{e-\mathrm{ph}}=\Sigma V\left(T_{e}^{5}-T_{\mathrm{ph}}^{5}\right),
$$

where $\Sigma=2 \times 10^{9} \mathrm{~W} \mathrm{~K}^{-5} \mathrm{~m}^{-3}$ for $\mathrm{Cu}, \mathcal{V}$ is the volume of the normal island, and $T_{e}$ and $T_{\mathrm{ph}}$ are the electron and phonon temperature, respectively.

Recently, we have developed a technique [25] to fabricate large-area $S-I-N-I-S$ coolers targeted at optimizing both $\dot{Q}_{e-\mathrm{ph}}$ and $\dot{Q}_{N I S}$. First, the cooled normal metal is suspended on top of the superconducting electrodes and, thus, quite decoupled from the substrate phonons. Second, hot quasiparticles in the leads are efficiently thermalized

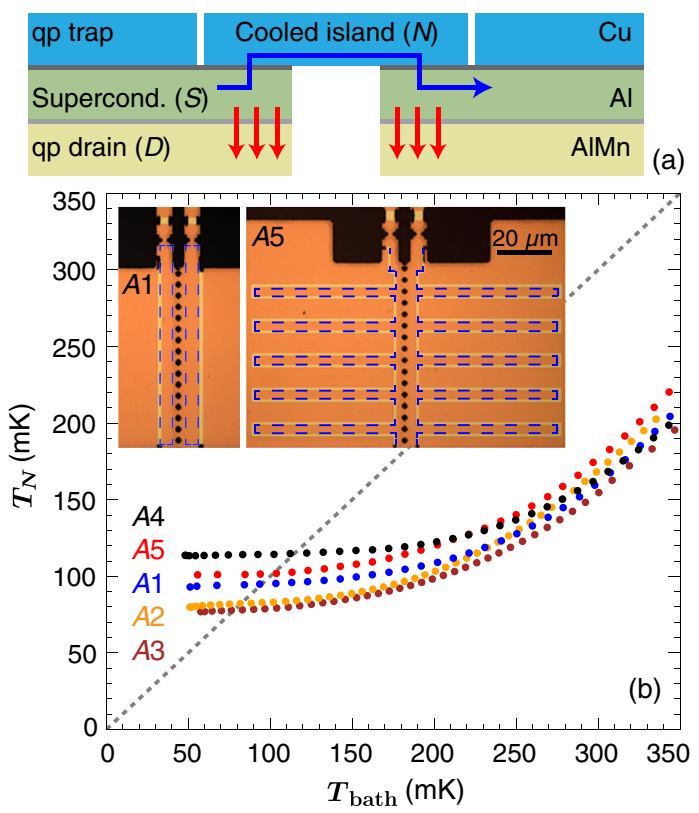

FIG. 1. (a) Schematic cross-sectional view of the $S-I-N-I-S$ cooler, where $\mathrm{Cu}$ is for the normal-metal island and quasiparticle traps, Al for the superconducting leads, and AlMn for the quasiparticle drains. The blue arrow indicates the charge current, and the red arrows show the heat current. (b) Normal-metal electronic temperature $T_{N}$ reached at the optimum bias vs bath temperature $T_{\text {bath }}$ for samples made on the same wafer but differing in their geometries. Compared to the standard sample $A 1\left(70 \times 4 \mu \mathrm{m}^{2}\right.$ for one junction), $A 2$ has twice the junction area, $A 3$ has half the junction area, and $A 4$ has $20 \%$ of its normal-metal volume. The left inset shows an image of the standard sample $A 1$, and the right inset shows an image of $A 5$ with an interdigitated junction shape. The $N-I-S$ junctions are bordered with dashed lines. with a normal-metal drain coupled to the superconductor through a transparent tunnel barrier [26].

In this paper, we show that these two advanced features, combined with an optimized tunnel junction transparency, improve the performance of a $S-I-N-I-S$ cooler significantly. At intermediate temperatures where electronphonon coupling is substantial, phonons in the suspended normal metal are cooled. At low temperatures, where the cooler is almost free from electron-phonon interaction, we tune the overheating in the superconducting leads by adjusting the transparency of the cooling junctions tunnel barrier. The most efficient $S-I-N-I-S$ cooler reaches about $30 \mathrm{mK}$, which is about $3 \%$ of $\Delta / k_{B}$. We discuss the heat transport and the benefit of having a quasiparticle drain coupled to the superconductor, as well as the possible reasons for the saturation at the lowest temperature.

\section{FABRICATION AND MEASUREMENT METHODS}

By following Ref. [25], the devices are fabricated by using photolithography and metal wet etch; see Fig. 1(a) for a schematic view and the inset in Fig. 1(b) for a top view of the samples. In this work, all coolers have a suspended normal metal bridging two 200-nm-thick $\mathrm{Al}$ superconducting electrodes sitting on top of a 200-nm-thick AlMn quasiparticle drain [26]. The $\mathrm{Al}$ and AlMn layers are separated by a thin $\mathrm{AlO}_{x}$ layer, oxidized in a mixture of Ar: $\mathrm{O}_{2}$, ratio $10: 1$ at pressure $2 \times 10^{-2} \mathrm{mbar}$ for $2 \mathrm{~min}$; see Table I for more sample parameters. All samples except $C 2$ (see below) have in addition (to the drain) a quasiparticle trap of $\mathrm{Cu}$ next to the junction. Coolers are measured with the standard four-probe technique in a dilution cryostat. The electronic temperature $T_{N}$ on the normal metal is

TABLE I. Parameters of the measured $S-I-N-I-S$ coolers. $P_{\mathrm{O}_{2}} / t_{\mathrm{O}_{2}}$ refers to the oxidation pressure and time used for producing the tunnel barrier of the cooler. $l \times w$ is the length $\times$ width of a rectangular $N-I-S$ junction. We write the area for $A 5$ in $\mu \mathrm{m}^{2}$, as it has an interdigitated shape. $d_{\mathrm{Cu}}$ is the thickness of the normal-metal island. $2 R_{T}$ is the tunnel resistance of the two $N-I-S$ cooling junctions in series. All samples gap $2 \Delta=375 \mu \mathrm{eV}$. "Figure" indicates the figure where the data on this sample are shown.

\begin{tabular}{lccccc}
\hline \hline Samples & $\begin{array}{c}P_{\mathrm{O}_{2}} / t_{\mathrm{O}_{2}} \\
(\mathrm{mbar} / \mathrm{min})\end{array}$ & $\begin{array}{c}l \times w \\
(\mu \mathrm{m} \times \mu \mathrm{m})\end{array}$ & $\begin{array}{c}d_{\mathrm{Cu}} \\
(\mathrm{nm})\end{array}$ & $\begin{array}{c}2 R_{T} \\
(\Omega)\end{array}$ & Figure \\
\hline$A 1$ & $1.3 / 5$ & $70 \times 4$ & 100 & 1.7 & $1,2,3$ \\
$A 2$ & $1.3 / 5$ & $70 \times 10$ & 100 & 0.75 & 1 \\
$A 3$ & $1.3 / 5$ & $70 \times 2$ & 100 & 3.4 & 1 \\
$A 4$ & $1.3 / 5$ & $70 \times 4$ & 20 & 1.2 & 1 \\
$A 5$ & $1.3 / 5$ & 950 & 100 & 0.8 & 1 \\
$B$ & $9 / 5$ & $70 \times 4$ & 60 & 3.7 & 2 \\
$C 1$ & $13 / 5$ & $70 \times 4$ & 60 & 4.8 & 2,3 \\
$C 2$ & $13 / 5$ & $70 \times 4$ & 60 & 4.6 & 2 \\
$D$ & $50 / 120$ & $70 \times 4$ & 60 & 10.5 & 2,3 \\
\hline \hline
\end{tabular}




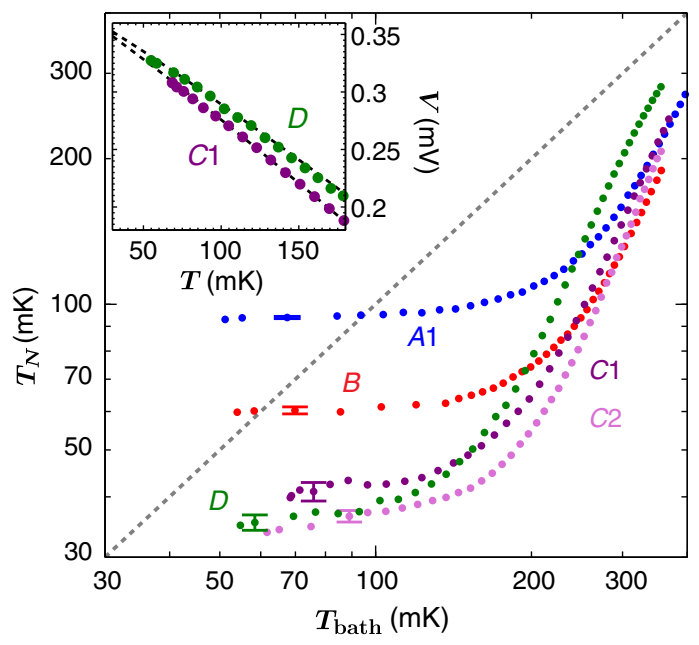

FIG. 2. Temperature of the normal-metal island $T_{N}$ at the optimum bias as a function of bath temperature $T_{\text {bath }}$. Samples $A 1, B, C 1$, and $D$ differ only in their tunnel resistances $R_{T}$ (Table I). $C 2$ is an improved version of $C 1$; see the text. The gray dotted line is the 1-1 line at the boundary between cooling and heating. The error bar for each data set from the measurement is represented. The inset shows thermometer calibrations, i.e., voltages of the probing junctions at a current of $1.5 \mathrm{nA}$ for samples $C 1$ and $D$ at cryostat temperatures below $200 \mathrm{mK}$. Dots are experiment data and dashed lines fit to the BCS theory.

measured by using a pair of smaller $N-I-S$ junctions [3], where the voltage drop under a constant current (about $1 \mathrm{nA}$ ) is calibrated to the cryostat temperature. Below the lowest cryostat temperature of $50 \mathrm{mK}$, the temperature $T_{N}$ is extracted based on an extrapolation of BCS theory using data from the higher-temperature regime. The measurement noise-related uncertainty is less than $300 \mu \mathrm{K}$; see Fig. 2 . Extracting the temperature from fitting the current-voltage characteristic to isothermal theory curves [20,22] gives identical results, including those below the lowest temperature of the cryostat where the difference is smaller than $1 \mathrm{mK}$ between the two methods.

\section{EXPERIMENTAL RESULTS}

Figure 1(b) demonstrates that, compared to the cooling power, electron-phonon interactions are negligible in our devices. Here, all coolers are made of the same $\mathrm{AlMn} / \mathrm{AlO}_{x} / \mathrm{Al} / \mathrm{AlO}_{x} / \mathrm{Cu}$ multilayer from a single wafer, but with a varying junction geometry. Taking $A 1$ as the reference sample, the $A 2$ junction has a double junction size [27] and $A 3$ half of that in $A 1 . A 4$ has the same junction size as $A 1$, but its $\mathrm{Cu}$ thickness is $20 \%$ of the value in $A 1$. The junction in sample $A 5$ has an interdigitated shape, where quasiparticle traps surround the $N-I-S$ junctions. As $\dot{Q}_{N I S} \propto$ $R_{T}^{-1}$ and $\dot{Q}_{e-\mathrm{ph}} \propto \mathcal{V}$, varying the junction size and the normal-metal volume can give information about the heat balance. Nonetheless, it is hard to see a real trend in the data, even near $300 \mathrm{mK}$, where $\dot{Q}_{e-\mathrm{ph}} \sim \Sigma \mathcal{V} T_{\mathrm{ph}}^{5}$ is expected to be significant [28]. We conclude that the present electronic coolers are almost free of electron-phonon interaction so that $\dot{Q}_{e \text {-ph }}$ is not responsible for the saturation of the normalmetal temperature $T_{N \text {,min }}$ in the low-temperature end. As a consequence, adjusting the normal-metal geometry does not improve its low-temperature performance.

Our main practical achievement is presented in Fig. 2: Starting from a $150-\mathrm{mK}$ bath temperature, the most powerful coolers reach a 30-mK electronic temperature, a fivefold reduction of temperature. Samples $A 1, B, C 1$, and $D$ are made by using an identical recipe and differ only in the cooler barrier resistance $2 R_{T}$ with respective values 1.7 , 3.7, 4.8, and $10.5 \Omega$. A smaller $R_{T}$ leads to a larger cooling power, which is beneficial. Nevertheless, it also leads to a stronger quasiparticle injection, which overheats the superconducting leads and degrades cooling at low temperature. Adjusting the tunnel resistance $R_{T}$ is therefore essential for optimizing electronic cooling. Within our sample set, sample $A 1$ with a large cooling power works best near $300 \mathrm{mK}$ but saturates at $94 \mathrm{mK}$. Sample $B$ reaches a lower temperature of $60 \mathrm{mK}$ thanks to a smaller dissipation by the injection current. Sample $D$ has the lowest cooling power at high temperature but cools down to $32 \mathrm{mK}$. Sample $C 1$ is a compromise between $B$ and $D$ and performs well over a wide temperature range. We conclude that the higher cooling power that is desirable at high temperatures compromises the performance of the device at the lowtemperature end, due to the backflow of heat from the overheated leads.

In order to investigate the limits of electronic cooling in the samples, we have improved sample $C 1$ in a number of ways, so that it is afterwards called $C 2$. First, $C 2$ is equipped with a pair of direct quasiparticle traps, which locate $1 \mu \mathrm{m}$ away from the junction. The superconducting leads are then affected by the direct contact with the normal metal and are thus expected to conduct heat better $[23,24]$. This modification would improve the performance of $C 2$ if the quasiparticle drain were limiting the performance in $C 1$. Second, we bond sample $C 2$ with $\mathrm{Au}$ wires, which have a much higher thermal conductance as compared to the usual superconducting $\mathrm{Al}$ wires employed in other samples. Finally, we measure sample $C 2$ in a rf-tight double-shielded sample stage [29]. These improvements work toward eliminating extra heating from quasiparticles and phonons as well as the radiation from the high-temperature parts of the cryostat. Despite all of the effort, sample $C 2$ performs only slightly better than $C 1$ in the whole temperature range covered. This result implies that the drain constitutes by itself an efficient quasiparticle trap that crucially helps an electronic cooler to reach $30 \mathrm{mK}$.

Let us now compare the cooler performance to theoretical predictions. We first assume a good thermalization of the metal phonons to the bath temperature: $T_{\mathrm{ph}}=T_{\text {bath }}$. Figure 3(a) shows the efficiency $\dot{Q}_{e \text {-ph }} / I V$ calculated within this framework for samples $A 1, C 1$, and $D$ at the optimum 


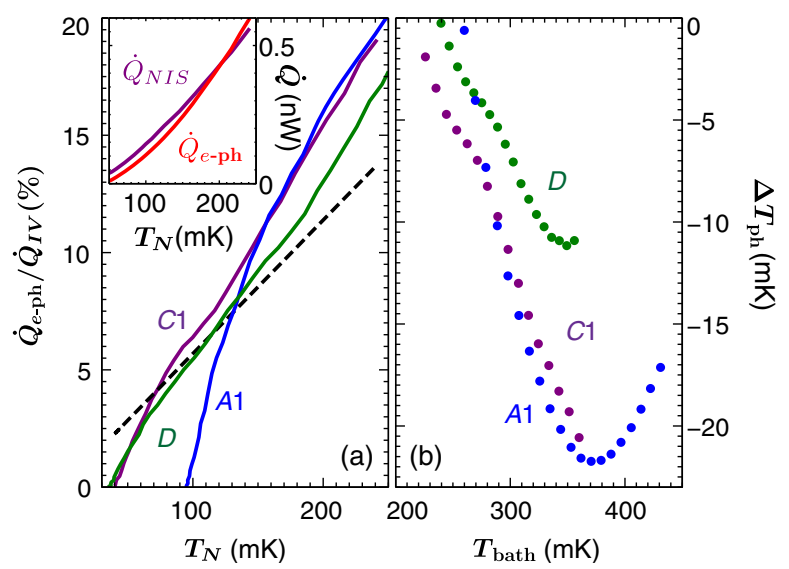

FIG. 3. (a) Apparent efficiency calculated with the assumption of metal phonons thermalized at the bath temperature for samples $A 1, C 1$, and $D$ compared to the theory prediction Eq. (2) (black dashed line). The inset shows the calculated $\dot{Q}_{N I S}$ when assuming $T_{S}=T_{\text {bath }}$ and $\dot{Q}_{e \text {-ph }}$ when assuming $T_{\text {ph }}=T_{\text {bath }}$ for sample $C 1$. (b) Extracted phonon temperature of the normal island $\Delta T_{\mathrm{ph}}=$ $T_{\text {ph }}-T_{\text {bath }}$ assuming the theoretical efficiency and no overheating of the leads.

cooling point. The measured quantity exceeds the prediction of Eq. (2) over a wide temperature range. In the inset, the comparison of the $N-I-S$ cooling power Eq. (1) with the electron-phonon coupling power Eq. (3) confirms this conclusion for sample $C 1$ : The two curves cross near $T_{N}=220 \mathrm{mK}$, above which $\dot{Q}_{e-\mathrm{ph}}>\dot{Q}_{N I S}$. Here we assumed no overheating in the superconductor: $T_{S}=$ $T_{\text {bath }}$ in Eq. (1), which gives an upper estimate for $\dot{Q}_{N I S}$. As the excess efficiency occurs at intermediate temperatures near $T_{\text {bath }}=300 \mathrm{mK}$, it is best explained by assuming that not only the electrons but also the phonons of the normal island cool, i.e., $T_{\mathrm{ph}}<T_{\text {bath. }}$. Our samples are particularly suited for this cooling to take place, as the normal-metal island is suspended on the superconducting electrodes and thus decoupled from the substrate. In order to estimate the phonon cooling, we calculate the drop in phonon temperature $\Delta T_{\mathrm{ph}}=T_{\text {bath }}-T_{\mathrm{ph}}$ necessary to fulfill the heat balance Eq. (2); see Fig. 3(b). A phonon cooling of about $20 \mathrm{mK}$ is obtained in samples $A 1$ and $C 1$. Again, the probable overestimation of $\dot{Q}_{N I S}$ makes the present phonon-temperature drop estimation a minimum value.

\section{ANALYSIS OF THE RESULTS AND DISCUSSION}

To illustrate the benefit of having an efficient quasiparticle drain, we now analyze the heat transport by using a diffusion model sketched in Fig. 4(a). In the $y$ direction, the temperature change $\delta T_{S}(y)$ across the superconductor thickness $d_{S}$ is estimated to be $\frac{y \dot{Q}_{\text {in }}}{A \kappa_{S}}$, where $\kappa_{S}$ is the electronic thermal conductivity of the superconductor and $\dot{Q}_{\text {in }}$ is the input power from the cooler barrier of area $A$. The
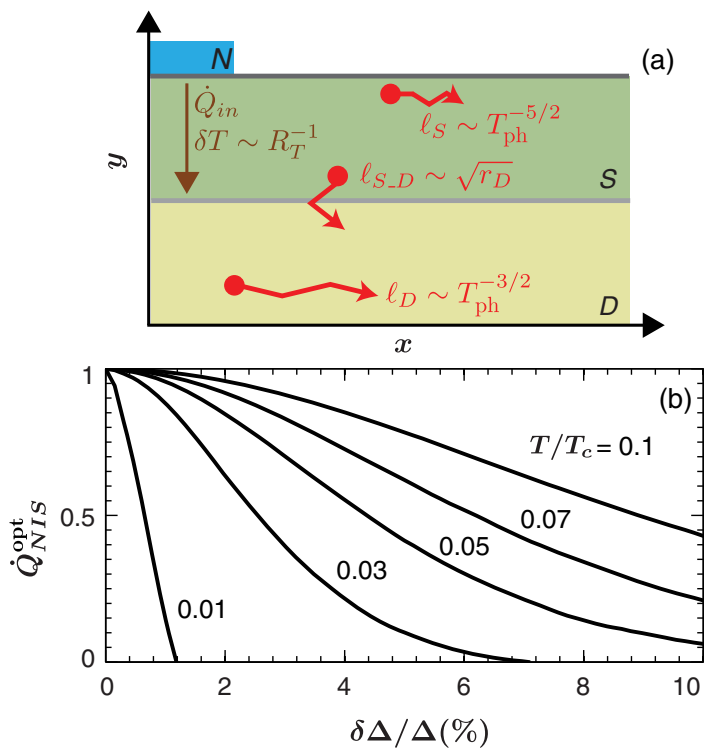

FIG. 4. (a) Sketch of the thermal transport when the superconductor couples to the quasiparticle drain. The red arrows represent the heat current where quasiparticles relax. We label their most notable dependences; see the text. (b) Optimum cooling power normalized by that of an ideal uniform junction as a function of the gap nonuniformity at different temperatures.

temperature drop is only $\delta T_{S}=0.4 \mathrm{mK}$ under a $1-\mathrm{nW}$ input power at $250 \mathrm{mK}$ and, thus, negligible. Along the $x$ direction, we use a thermal conductivity $\kappa_{D}=\mathcal{L}_{0} \rho_{D}^{-1} T_{D}$ in the normal-metal drain, where $\mathcal{L}_{0}$ is the Lorenz number, $T_{D}$ the local temperature, and $\rho_{D}$ the resistivity. The density of the heat current is $\dot{q}_{D}=\Sigma_{D}\left(T_{D}^{5}-T_{\mathrm{ph}}^{5}\right)$, where $\Sigma_{D}$ is the electron-phonon coupling in the drain material. For a small temperature change, we obtain a heat relaxation length $\ell_{D}=\sqrt{\mathcal{L}_{0} / 5 \rho_{D} \Sigma_{D}} T_{\mathrm{ph}}^{-3 / 2}$. For AlMn, this yields $\ell_{D}=$ $11 T^{-3 / 2} \mu \mathrm{m} \mathrm{K}^{-3 / 2}(120 \mu \mathrm{m}$ at $200 \mathrm{mK})$ [30]. In the superconducting leads, the heat conductivity is decreased by a factor of $6\left(\frac{\Delta}{k_{B} T_{S}}\right)^{2} \exp \left(-\Delta / k_{B} T_{S}\right)$, while the heat exchange is reduced by $\exp \left(-\Delta / k_{B} T_{S}\right)$ [31]. We obtain $\ell_{S}=\frac{\Delta}{\pi k_{B}} \sqrt{6 \mathcal{L}_{0} / 5 \rho_{S} \Sigma_{S}} T_{\mathrm{ph}}^{-5 / 2}$. For pure Al without a trap, $\ell_{S}=50 T^{-5 / 2} \mu \mathrm{m} \mathrm{K}^{-5 / 2}$ (3 $\mathrm{mm}$ at $200 \mathrm{mK}$ ). The heat transport along the superconducting leads is thus much less efficient than in the drain, as expected.

When the superconductor couples to the drain, the heat flux through the barrier in between is given by [32]

$$
\dot{q}=\frac{\sqrt{2 \pi k_{B} \Delta^{3}}}{e^{2} d_{S} r_{D}}\left(\sqrt{T_{S}} e^{-\Delta / k_{B} T_{S}}-\sqrt{T_{D}} e^{-\Delta / k_{B} T_{D}}\right),
$$

where $r_{D}$ is the junction resistance per area. The relaxation length in the $x$ direction is then written

$$
\ell_{S_{-} D}=\left(\frac{r_{D} d_{S}}{\rho_{S}} \sqrt{\frac{8}{\pi} \frac{k_{B} T_{D}}{\Delta}}\right)^{1 / 2}=2 \sqrt{r_{D}} \Omega^{1 / 2} \mu \mathrm{m}
$$


at $300 \mathrm{mK}$. A small specific resistance $r_{D}$ is thus needed so that the superconductor is locally efficiently thermalized to the drain. The similar cooling behavior of sample $C 2$ with a direct quasiparticle trap and the original sample $C 1$ leads to the conclusion that heat relaxation in $C 1$ or $C 2$ occurs at a distance from the junction smaller than the distance of the direct trap, i.e., $\ell_{S_{-} D} \lesssim 5 \mu \mathrm{m}$. This statement leads to the estimate $r_{D} \lesssim 10 \Omega \mu \mathrm{m}^{2}$, which is consistent with expectations based on the fabrication recipe and with a previous evaluation [26].

Let us now estimate the different terms in the heat balance at the lowest temperature range. We consider sample $C 1$ at its lowest bath temperature of $70 \mathrm{mK}$, assuming $T_{S}=T_{\mathrm{ph}}=T_{\text {bath }}$. Joule heating on the normal island, with a resistance of $0.02 \Omega$ and a typical $1-\mu \mathrm{A}$ injection current, is only $20 \mathrm{fW}$ and thus negligible. The electron-phonon coupling power in the $\mathrm{Cu}$ island $\dot{Q}_{e \text {-ph }}=$ $0.17 \mathrm{pW}$ is small in comparison to the cooling power $\dot{Q}_{N I S}=20 \mathrm{pW}$. The phenomenological Dynes factor $\gamma=$ $G_{0} / G_{N}$ is the ratio of the conductance at zero bias to its normal-state value. It captures the contribution of possible pin holes in the tunnel barrier, the inverse proximity effect in the superconductor, and the effect of the environment on electron tunneling. The fit of the current-voltage characteristics (data not shown) gives $\gamma=2.5 \times 10^{-4}$, which brings a related parasitic heating power $\Delta^{2} \gamma / R_{T} \simeq 8 \mathrm{pW}$. As $T_{N, \text { min }} \simeq 2.5 \gamma^{2 / 3} T_{c}$ [4], the observed minimum temperature $T_{N \text {, min }} \simeq 30 \mathrm{mK}$ could be related to a $\gamma$ parameter at least 3 times larger than the measured value. Thus, the Dynes smearing of the superconductor density of states cannot account for the observed temperature saturation.

The discrepancy in the power balance necessarily comes from other sources that we do not have a direct way to probe. Besides potential candidates such as phonon heating [33] or near-field heat transport [34], we also suspect that the nonuniformity of the superconducting gap contributes to the saturation of $T_{N, \min }$. It is well known that the superconducting gap $\Delta$ of a thin $\mathrm{Al}$ film can have different values depending on the fabrication details. The gap can be tuned by the grain size of the film $[35,36]$, and different crystal orientations can have a different value up to $3 \%$ [37,38]. To model the performance of the cooler, we assume a Gaussian distribution of $\Delta$ with a standard deviation $\delta \Delta$ and calculate the maximum cooling power $\dot{Q}_{N I S}^{\text {opt }}$ normalized to its value at $\delta \Delta=0$. Figure 4(b) shows our result at different temperatures. With $\delta \Delta / \Delta=3 \%$ at $30 \mathrm{mK}\left(T / T_{c}=0.03\right)$, the cooling power would be reduced to one-half of its nominal value.

\section{CONCLUSIONS}

In conclusion, the accumulation of hot quasiparticles in the superconducting leads limits the cooler performance, even with an efficient quasiparticle drain. We reduce this effect by tuning the tunnel barrier and demonstrate that such a cooler reaches a 32-mK electron temperature from a $150-\mathrm{mK}$ bath temperature and performs outstandingly over a wide range of temperatures. At temperatures where electron-phonon interaction is strong, phonon cooling in the suspended normal-metal island boosts the performance of the cooler. This refrigerator has a significant power and can be easily integrated, so that it opens new possibilities to cool practical devices to the sub-50-mK regime.

\section{ACKNOWLEDGMENTS}

We have benefited from discussions with D. V. Averin, Y. A. Pashkin, D. S. Golubev, M. Prunnila, V. F. Maisi, I. Khaymovich, T. T. Heikkilä, V. J. Kauppila, and C. B. Winkelmann. We acknowledge the support of the European Community Research Infrastructures under the FP7 Capacities Specific Program, MICROKELVIN Project No. 228464, the Engineering and Physical Sciences Research Council (EPSRC) Grant No. EP/F040784/1, the Academy of Finland through its LTQ CoE grant (Project No. 250280 and Project No. 139172), and the OtaNano infrastructure. Samples are fabricated in the Micronova Nanofabrication Center of Aalto University.

[1] M. Nahum, T.M. Eiles, and J.M. Martinis, Electronic microrefrigerator based on a normal-insulatorsuperconductor tunnel junction, Appl. Phys. Lett. 65, 3123 (1994).

[2] J. T. Muhonen, M. Meschke, and J. P. Pekola, Micrometrescale refrigerators, Rep. Prog. Phys. 75, 046501 (2012).

[3] F. Giazotto, T. T. Heikkilä, A. Luukanen, A. M. Savin, and J. P. Pekola, Opportunities for mesoscopics in thermometry and refrigeration: Physics and applications, Rev. Mod. Phys. 78, 217 (2006).

[4] J. P. Pekola, T. T. Heikkilä, A. M. Savin, J. T. Flyktman, F. Giazotto, and F. W. J. Hekking, Limitations in cooling electrons using normal-metal-superconductor tunnel junctions, Phys. Rev. Lett. 92, 056804 (2004).

[5] M. M. Leivo, J. P. Pekola, and D. V. Averin, Efficient Peltier refrigeration by a pair of normal metal/insulator/ superconductor junctions, Appl. Phys. Lett. 68, 1996 (1996).

[6] A. S. Vasenko, E. V. Bezuglyi, H. Courtois, and F. W. J. Hekking, Electron cooling by diffusive normal metalsuperconductor tunnel junctions, Phys. Rev. B 81, 094513 (2010).

[7] P. J. Lowell, G. C. O’Neil, J. M. Underwood, and J. N. Ullom, A nanoscale refrigerator for macroscale objects, Appl. Phys. Lett. 102, 082601 (2013).

[8] N. A. Miller, G. C. O’Neil, J. A. Beall, G. C. Hilton, K. D. Irwin, D. R. Schmidt, L. R. Vale, and J. N. Ullom, High resolution $\mathrm{X}$-ray transition-edge sensor cooled by tunnel junction refrigerators, Appl. Phys. Lett. 92, 163501 (2008).

[9] Cryogenic Particle Detection, edited by Ch. Enss (Springer, New York, 2005).

[10] CDMS II Collaboration, Dark matter search results from the CDMS II experiment, Science 327, 1619 (2010). 
[11] S. M. Bilenky and C. Giunti, Neutrinoless double-beta decay: A brief review, Mod. Phys. Lett. A 27, 1230015 (2012).

[12] BICEP2 Collaboration, Detection of B-mode polarization at degree angular scales by BICEP2, Phys. Rev. Lett. 112, 241101 (2014).

[13] J. A. Tauber et al., Planck pre-launch status: Design and description of the Low Frequency Instrument, Astron. Astrophys. 520, A1 (2010).

[14] A. D. OConnell, M. Hofheinz, M. Ansmann, R. C. Bialczak, M. Lenander, E. Lucero, M. Neeley, D. Sank, H. Wang, M. Weides, J. Wenner, J. M. Martinis, and A. N. Cleland, Quantum ground state and single-phonon control of a mechanical resonator, Nature (London) 464, 697 (2010).

[15] J. M. Pirkkalainen, S. U. Cho, J. Li, G. S. Paraoanu, P. J. Hakonen, and M. A. Sillanpää, Hybrid circuit cavity quantum electrodynamics with a micromechanical resonator, Nature (London) 494, 211 (2013).

[16] J. P. Pekola, O.-P. Saira, V. F. Maisi, A. Kemppinen, M. Möttönen, Y. A. Pashkin, and D. V. Averin, Single-electron current sources: Toward a refined definition of the ampere, Rev. Mod. Phys. 85, 1421 (2013).

[17] F. Giazotto, J. T. Peltonen, M. Meschke, and J. P. Pekola, Superconducting quantum interference proximity transistor, Nat. Phys. 6, 254 (2010).

[18] P. J. Koppinen and I. J. Maasilta, Phonon cooling of nanomechanical beams with tunnel junctions, Phys. Rev. Lett. 102, 165502 (2009).

[19] L. M. A. Pascal, A. Fay, C. B. Winkelmann, and H. Courtois, Existence of an independent phonon bath in a quantum device, Phys. Rev. B 88, 100502 (2013).

[20] S. Rajauria, P. S. Luo, T. Fournier, F. W. J. Hekking, H. Courtois, and B. Pannetier, Electron and phonon cooling in a superconductor-normal metal-superconductor tunnel junction, Phys. Rev. Lett. 99, 047004 (2007).

[21] C. Wang, Y. Y. Gao, I. M. Pop, U. Vool, C. Axline, T. Brecht, R. W. Heeres, L. Frunzio, M. H. Devoret, G. Catelani, L. I. Glazman, and R. J. Schoelkopf, Measurement and control of quasiparticle dynamics in a superconducting qubit, arXiv:1406.7300.

[22] G. C. O’Neil, P. J. Lowell, J. M. Underwood, and J. N. Ullom, Measurement and modeling of a large-area normal-metal/insulator/superconductor refrigerator with improved cooling, Phys. Rev. B 85, 134504 (2012).

[23] A. Luukanen, A. M. Savin, T. I. Suppula, J. P. Pekola, M. Prunnila, and J. Ahopelto, Integrated SINIS refrigerators for efficient cooling of cryogenic detectors, AIP Conf. Proc. 605, 375 (2002).

[24] N. A. Court, A. J. Ferguson, R. Lutchyn, and R. G. Clark, Quantitative study of quasiparticle traps using the singleCooper-pair transistor, Phys. Rev. B 77, 100501 (2008).
[25] H. Q. Nguyen, L. M. A. Pascal, Z. H. Peng, O. Buisson, B. Gilles, C. B. Winkelmann, and H. Courtois, Etching suspended superconducting tunnel junctions from a multilayer, Appl. Phys. Lett. 100, 252602 (2012).

[26] H. Q. Nguyen, T. Aref, V. J. Kauppila, M. Meschke, C. B. Winkelmann, H. Courtois, and J. P. Pekola, Trapping hot quasi-particles in a high-power superconducting electronic cooler, New J. Phys. 15, 085013 (2013).

[27] Because of the large junction size, $A 2$ has 1-nW cooling power at $300 \mathrm{mK}$. Still, it performs equally well compared to $A 1$ or $A 3$.

[28] The crossing of data from sample $A 4$ with those from other samples is vaguely inline with expectation, as $A 4$ has the smallest metal volume $\mathcal{V}$. Its higher $T_{N \text {,min }}$ is presumably due to Joule heating because of the thinner $\mathrm{Cu}$ layer.

[29] O.-P. Saira, A. Kemppinen, V. F. Maisi, and J. P. Pekola, Vanishing quasiparticle density in a hybrid $\mathrm{Al} / \mathrm{Cu} / \mathrm{Al}$ single-electron transistor, Phys. Rev. B 85, 012504 (2012).

[30] Because of the weak dependence $\ell_{D} \propto 1 / \sqrt{\Sigma_{D} \rho_{D}}$, the corresponding numbers for $\mathrm{Cu}$ and $\mathrm{Au}$ are similar. There is no advantage of replacing AlMn by another material.

[31] A. V. Timofeev, C. Pascual Garcia, N. B. Kopnin, A. M. Savin, M. Meschke, F. Giazotto, and J. P. Pekola, Recombination-limited energy relaxation in a BardeenCooper-Schrieffer superconductor, Phys. Rev. Lett. 102, 017003 (2009).

[32] J. T. Peltonen, J. T. Muhonen, M. Meschke, N. B. Kopnin, and J.P. Pekola, Magnetic-field-induced stabilization of non-equilibrium superconductivity in a normal-metal/ insulator/superconductor junction, Phys. Rev. B 84, 220502 (2011).

[33] L. M. A. Pascal, H. Courtois, and F. W. J. Hekking, Circuit approach to photonic heat transport, Phys. Rev. B 83, 125113 (2011).

[34] S. Biehs, P. Ben-Abdallah, and F. S. S. Rosa, in Infrared Radiation, Vol. 1, edited by V. Morozhenko (InTech, Rijeka, Croatia, 2012).

[35] T. Yamamoto, Y. Nakamura, Y. A. Pashkin, O. Astafiev, and J. S. Tsai, Parity effect in superconducting aluminum single electron transistors with spatial gap profile controlled by film thickness, Appl. Phys. Lett. 88, 212509 (2006).

[36] J. Aumentado, M. W. Keller, J. M. Martinis, and M. H. Devoret, Non-equilibrium quasiparticles and 2e periodicity in single-Cooper-pair transistors, Phys. Rev. Lett. 92, 066802 (2004).

[37] G. L. Wells, J. E. Jackson, and E. N. Mitchell, Superconducting tunnelling in single-crystal and polycrystal films of aluminum, Phys. Rev. B 1, 3636 (1970).

[38] M. F. Biondi, M. P. Garfunkel, and W. A. Thompson, Millimeter-microwave studies of energy gap anisotropy in superconductors, Phys. Rev. 136, A1471 (1964). 